\title{
Audio AR to Support Nature Connectedness in People with Visual Disabilities
}

\author{
Maryam Bandukda \\ PhD Student \\ UCL Interaction Centre \\ Global Disability Innovation Hub \\ University College London United Kingdom \\ m.bandukda@ucl.ac.uk
}

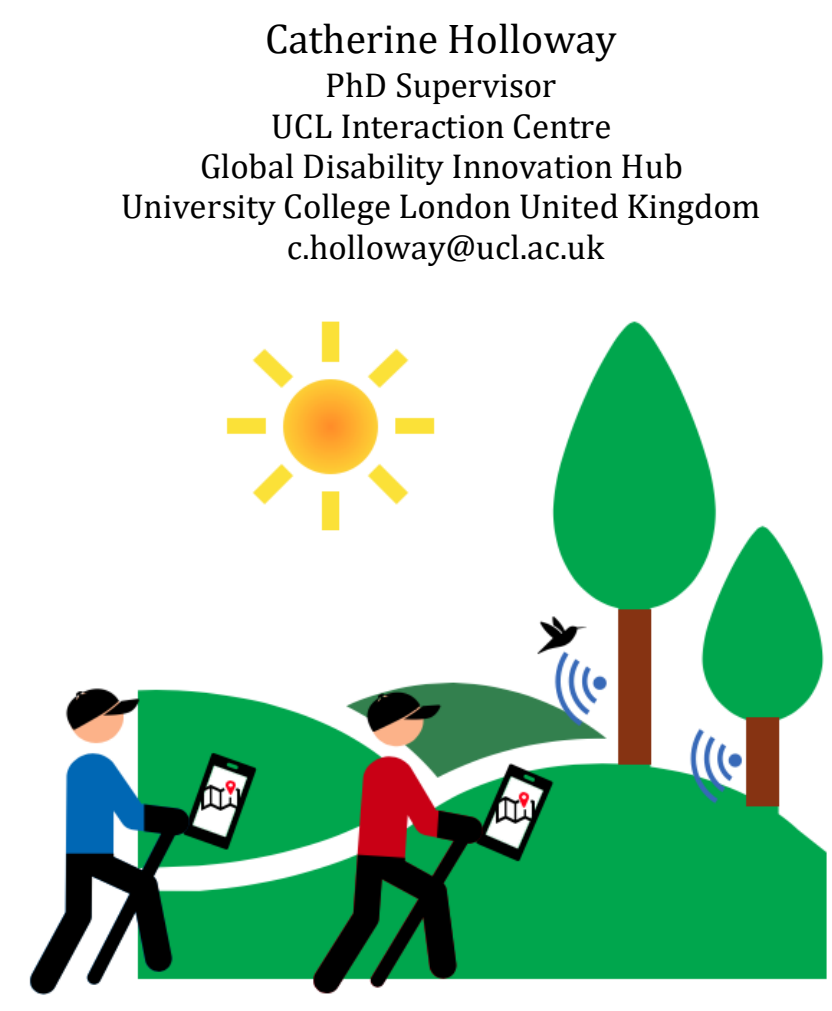

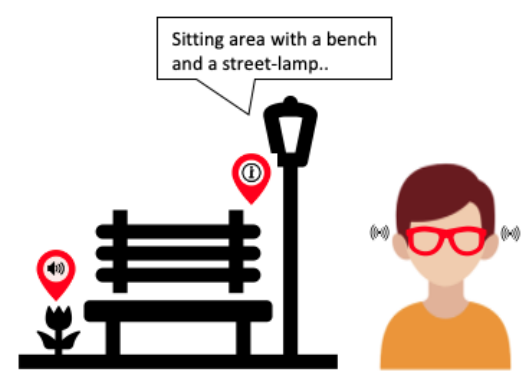

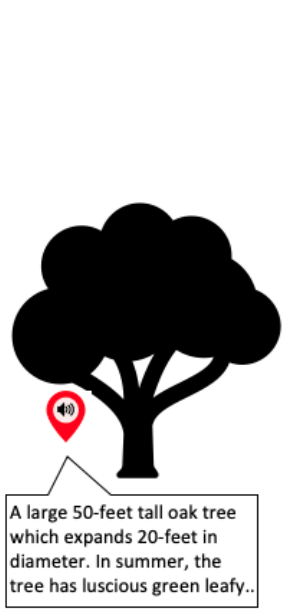

Figure 1: An auditory augmented reality system to support blind and partially sighted people in exploring open spaces and connect with nature by augmenting spatial and natural elements with audio and sonification. This system encourages multisensory exploration through non-visual interaction with technology and the physical environment.

\begin{abstract}
Nature and outdoor open spaces are good for our mental and physical health; providing space for exercise, relaxation, socializing and exploring nature. Technology plays an important role in how people explore the outdoors, however, despite the prevalence of mobile technologies that promote outdoor mobility, they are often not accessible to people with disabilities. This $\mathrm{PhD}$ project explores technologies to promote nature connectedness in blind and partially sighted people. We have conducted formative studies exploring the needs of blind and partially sighted people and barriers that limit their experiences. The next phase of my research will focus on designing auditory augmented reality systems to augment the natural elements in open spaces which are presented to the user in real-time as they navigate the space. We aim to design, implement, and evaluate pervasive auditory augmented reality systems that enhance people's immersive experience and engagement with nature.
\end{abstract}

\section{CCS CONCEPTS}

-Human-centred computing Accessibility Accessibility technologies $\bullet$ Human-centred computing $\sim$ Human computer interaction $(\mathrm{HCI}) \sim$ Interaction paradigms $\sim$ Mixed / augmented reality

\section{KEYWORDS}

Nature, auditory augmented reality, accessibility, blind and partially sighted, spatial audio

\section{ACM Reference Format:}

TBD

\section{PROBLEM STATEMENT}

An estimated 285 million people live with visual impairment worldwide, of which 39 million are blind [20]. In the UK, 2 million people living with visual impairment of which approx. 330,000 are registered blind [13]. The rate of prevalence of visual impairment and blindness is increasing. It is estimated that the number of people living with sight loss worldwide will triple due to population growth and ageing. $[\underline{14}, \underline{19}]$.

Loss of vision affects people's quality of life by limiting their ability to manage their daily activities. The impact of sight loss on people's lives varies based on the severity of sight loss and 
the age of onset but mostly impacts the individual's independence.

Open spaces such as parks are great for exploration, relaxation, socializing, and physical activity $[5,6,12]$. These spaces allow nature connectedness through sensory and emotional engagement and promote nature conservation. Blind and partially sighted people (BPSP) are often unable to fully enjoy their outdoor experience and connect with nature due to limited vision. This makes it difficult for BPSP young people to develop mental maps of open spaces and to explore the natural elements. For many people who lose sight later in life, sight loss means being unable to continue enjoying nature.

Although AR/VR systems in recent years have focused on improving outdoor mobility for BPSP $[10,23]$, there exists a gap in research on AR accessibility for leisure and engaging with nature. Furthermore, a majority of the accessible AR/VR systems have been designed to be used in lab-based settings. Our research aims to extend the current research on auditory augmented reality (AAR) by designing, implementing, and evaluating in-situ an accessible AAR system for BPSP.

\section{$1.1 \quad$ Research questions}

Our research takes a user-centred approach to design and comprises of two main research phases. In phase 1, we conducted qualitative studies to develop an in-depth understanding of the needs of BPSP in outdoor mobility and how they engage with nature. We have completed this phase and have partially published our findings $[\underline{3}, \underline{4}]$. In the next phase, we aim to design an AAR system to support BPSP in exploring open spaces and connect with nature. Table 1 below lists the research questions we addressed in phase 1 and aim to address in phase 2 .

\section{RELATED WORK}

Recent research has explored the uses of video and auditory AR systems for people with visual disabilities, including reading [18] and navigation $[\underline{1}, \underline{16}]$. Like video, AAR superimposes the environmental sounds with additional auditory information about the space. Microsoft Soundscape is an example of Spatial AAR systems that allow users to tag georeferenced virtual beacons or landmarks in the physical environment and attach additional information to such landmarks. The app uses localized audio to indicate the location of landmarks to the user when navigating a route. Footnotes [8] extends the geo-referenced information available in Microsoft Soundscape by adding visual, historical, social, and functional contextual information to the physical objects on the map. The spatial audio enabled BPSP to discover new landmarks and thoroughly explore new locations. Personalized audio feedback to enhance spatial awareness and navigation experience has been explored in many other studies. Albrecht [2] used spatialized music to guide pedestrians and cyclists along a route. [22] have investigated the use of directional audio to synthesize arbitrary sounds of objects in an indoor environment.

Table 1: Research questions

\begin{tabular}{c|c} 
RESEARCH QUESTIONS \\
RQ1.1: How do BPSP: (a) plan for visits to outdoor \\
natural spaces? (b) access these spaces? (c) \\
engage with the environment? \\
RQ1.2: How do the currently available technologies \\
facilitate these experiences? \\
RQ2.1: (a) How can accurate information about \\
natural elements in open spaces be collected? (b) \\
How can this information be accurately \\
presented to BPSP in-situ? \\
RQ2.2: (a) How effective can AAR systems be in \\
enhancing spatial understanding of BPSP and \\
supported nature connectedness? (b) Should \\
other modalities be included to make the \\
interaction more effective? \\
RQ2.3: (a)How can such a system encourage social \\
interaction between BPSP and non-BPSP while \\
supporting nature connectedness? (b) How can \\
such a system be made accessible for BPSP to \\
operate independently?
\end{tabular}

\section{RESEARCH APPROACH AND METHODS}

We have completed phase 1 (see Table 1) of this project, focusing on understanding the needs of BPSP in outdoor open spaces. In phase 2, we aim to design, implement, and evaluate an AAR system prototype to enhance spatial understanding of BPSP and encourage nature connectedness.

\subsection{Research carried out so far}

We have conducted formative qualitative studies including interviews, focus group, and qualitative survey questionnaire to examine the needs of BPSP when in open spaces (RQ1.1 and RQ1.2) $[3,4]$. We found that (1) verbal descriptions of the environment in addition to multisensory experience help BPSP explore and connect with nature (Figure 1), (2) social interaction was important and BPSP enjoyed co-creating a shared sensory experience with their companions. These findings led us to further explore 'auditory' experience and how it can be used to encourage nature connection for BPSP. 


\subsection{Planned work}

Building on the previous work, we aim to;

- $\quad$ Create a database of geo-tagged information about natural elements in parks and other natural space through crowdsourcing (RQ2.1a).

- $\quad$ Explore existing technologies that allow delivering the information in-situ and design an AAR system to present this information to users in-situ (RQ2.1b)

- Evaluate how an AAR system can enhance the spatial understanding and nature connectedness for BPSP (RQ2.2a)

- $\quad$ Explore the use of additional modalities such as haptics [2] , spatialized sound [2] to encourage exploration and make the experience more engaging (RQ2.2b)

- $\quad$ Explore the design approach to embed social elements within the AAR system to encourage social interaction between BPSP and companions (RQ2.3). Co-located social AR interactions have been explored to enhance social play and interaction with the physical environment [9,21] while promoting physical activity, exploration and collaboration $[2,11,15,17]$.

\section{THE AAR SYSTEM CONCEPT}
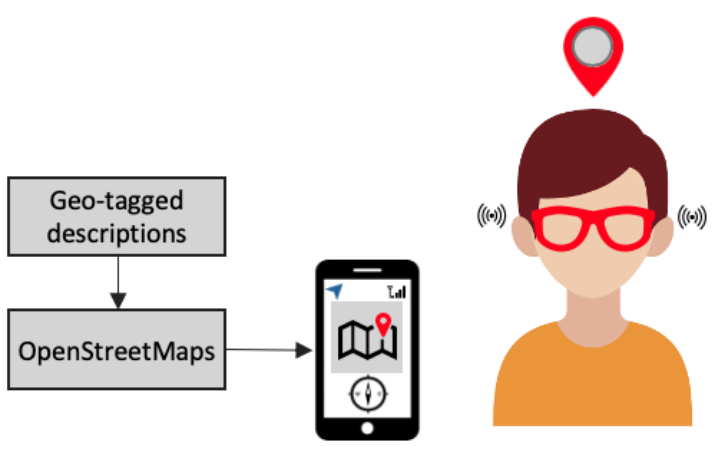

Figure 2: The AAR System Concept

We aim to design, implement, and evaluate an AAR system (shown in Figure 2) consisting of geo-tagged descriptions of landmarks (natural, infrastructural elements) in open spaces. These descriptions are called out to the user as they explore open space and are localized to the location of the landmark relative to the user; creating an immersive $360^{\circ}$ auditory experience. Given that hearing is crucial for BPSP to maintain spatial awareness, we aim to use commercially available headphones that allow spatial sound augmentation or 'transparent hearing' (e.g. Sennheiser Ambeo smart headset1 contains omnidirectional microphones that capture spatial sounds while listening to audio). Alternatively, off the shelf bone conduction headsets can also be used to enable the audio augmentation without blocking spatial sounds.

\section{EVALUATION APPROACH}

To evaluate the design and implementation of our AAR system, we will conduct user studies with BPSP. The user studies will be conducted in-situ at parks where the participants will explore the open space while using the AAR system. The user studies will evaluate:

- $\quad$ Spatial understanding: How localized spatial AAR can enhance the spatial understanding of BPSP in opens spaces. We will evaluate this by conducting pre and post-test survey with participants to gauge their understanding of the environment. The user study will involve the participant exploring the open space while using the AAR system. In a follow-up task, participants will be asked to recall different landmarks and locations to recreate the environment from their understanding. We will use a 5-point Likert scale to measure how confident participants feel in exploring the environment while using the AAR.

- Nature connectedness: How augmenting the natural environment with contextual descriptions [8] can improve nature connectedness of BPSP. Our findings from previous work $[3,4]$ show that BPSP enjoy learning about and engaging with the natural elements in open spaces. We will measure how localized audio will encourage engaging with nature by measuring the number of interactions with the environment (i.e. stopping to smell, touch, and listen to the natural elements) and a post-test survey to collect subjective responses. A follow up longitudinal study could be conducted to capture the long-term effect of nature connectedness and how that encourages subsequent visits to open spaces, however, it might be out of the scope of our project.

- Social interaction: Social interaction emerged as one of the key elements of meaningful experience in nature. Therefore, the AAR system would not only encourage BPSP to interact with the physical environment but also interact with their companions. We aim to integrate social interaction within the AAR system that would allow participants to share the soundscape with people around them.

\section{EXPECTED CONTRIBUTION TO UBIQUITOUS COMPUTING}

The proposed research sits at the intersection of accessibility and ubiquitous computing. The first phase of our research aims to contribute to $\mathrm{HCI}$ and accessibility literature, an in-depth understanding of the experiences of BPSP in open 
spaces including the needs, barriers and affective interaction (emotional and sensory experiences) with the environment. This will be a valuable contribution to HCI research and useful for the design of assistive technologies for BPSP. The second phase of our research proposes to contribute the design of an AAR system for enhanced nature exploration and connectedness. As there are currently no technologies that support nature connectedness for BPSPs, the design of AAR as part of this thesis will be a novel contribution to HCI research.

\section{AUTHOR BIOGRAPHY}

Maryam Bandukda is a third-year PhD student at UCL Interaction Centre and Global Disability Innovation Hub. Her $\mathrm{PhD}$ research is supervised by $\mathrm{Dr}$ Catherine Holloway, Prof. Nadia Berthouze, and Dr Aneesha Singh. Maryam started her $\mathrm{PhD}$ in September in 2017 and will complete by September 2021.

Maryam's research interests sit at the intersection of accessibility and augmented reality (AR). She is passionate about inclusivity and wellbeing. She aims to design AAR systems to support BPSP explore the natural environments and engage with nature. She has published her initial work at CHI as Late-Breaking Work and her recent work has been accepted at ASSETS 2020.

\section{ACKNOWLEDGMENTS}

This work is funded by EPSRC Doctoral Training Program EP/N509577/1. I am thankful to all the participants who have so far taken interest in my research and shared their valuable experience and insights with me. I'm also immensely grateful to my supervisors, Dr Catherine Holloway, Prof. Nadia Berthouze, and Dr Aneesha Singh for their continued support and guidance.

\section{REFERENCES}

1. Jérémy Albouys-Perrois, Jérémy Laviole, Carine Briant, and Anke Brock. 2018. Towards a Multisensory Augmented Reality Map for Blind and Low Vision People: a Participatory Design Approach. CHI'18 - CHI Conference on Human Factors in Computing Systems, ACM Press, 1-14.

2. Robert Albrecht, Riitta Väänänen, and - Tapio Lokki. Guided by music: pedestrian and cyclist navigation with route and beacon guidance. .

3. Maryam Bandukda, Catherine Holloway, Aneesha Singh, and Nadia BianchiBerthouze. 2020. PLACES : A Framework for Supporting Blind and Partially Sighted People in Outdoor Leisure Activities (In Press). ASSETS 2020 - 22nd International ACM SIGACCESS Conference on Computers and Accessibility.

4. Maryam Bandukda, Aneesha Singh, Nadia Berthouze, Catherine Holloway, Aneesha Singh, and Catherine Holloway. 2019. Understanding experiences of blind individuals in outdoor nature. Conference on Human Factors in Computing Systems - Proceedings, ACM Press, 1-6.

5. Ariane L. Bedimo-Rung, Andrew J. Mowen, and Deborah A. Cohen. 2005. The significance of parks to physical activity and public health: A conceptual model. American Journal of Preventive Medicine, Elsevier, 159-168.

6. Siân de Bell, Hilary Graham, Stuart Jarvis, and Piran White. 2017. The importance of nature in mediating social and psychological benefits associated with visits to freshwater blue space. Landscape and Urban Planning 167: 118127.

7. Steve Benford, Andy Crabtree, Martin Flintham, et al. 2006. Can You See Me
Now? .

8. Cole Gleason, Alexander J Fiannaca, Meredith Ringel Morris, Melanie Kneisel, and Edward Cutrell. 2018. FootNotes: Geo-referenced Audio Annotations for Nonvisual Exploration. Proc. ACM Interact. Mob. Wearable Ubiquitous Technol 2, 109: 24 .

9. Anhong Guo. 2019. Blocks: Collaborative and Persistent Augmented Reality Experiences. Proc. ACM Interact. Mob. Wearable Ubiquitous Technol 3, 3: 83.

10. O. Lahav and D. Mioduser. 2008. Haptic-feedback support for cognitive mapping of unknown spaces by people who are blind. International Journal of Human Computer Studies 66, 1: 23-35.

11. Susanna Paasovaara, Pradthana Jarusriboonchai, and Thomas Olsson. 2017. Understanding collocated social interaction between pokémon go players. ACM International Conference Proceeding Series, 151-163.

12. Scott A Paluska and Thomas L Schwenk. 2000. Physical aActivity and mental health. Sports Medicine 29, 3: 167-180.

13. RNIB. 2012. Key Information and Statistics. 2012, Sight Loss, Future Projections, Prevention, Cost o.

14. RNIB. 2012. Key Information and Statistics. 2012, Sight Loss, Future Projections, Prevention, Cost o. Retrieved from http://www.rnib.org.uk/aboutus/Research/statistics/Pages/statistics.aspx.

15. Y. Rogers, D. Stanton, M. Thompson, et al. 2004. Ambient wood. Proceeding of the 2004 conference on Interaction design and children building a community IDC '04, ACM Press, 3-10.

16. Marjan Sikora, Mladen Russo, Jurica Derek, and Ante Jurčević. 2018. Soundscape of an archaeological site recreated with audio augmented reality. ACM Transactions on Multimedia Computing, Communications and Applications 14, 3: 1-22.

17. Kiley Sobel, Arpita Bhattacharya, Alexis Hiniker, Jin Ha Lee, Julie A Kientz, and Jason C Yip. 2017. It wasn't really about the Pokémon: Parents' Perspectives on a Location-Based Mobile Game. Proceedings of the 2017 CHI Conference on Human Factors in Computing Systems - CHI '17, 1483-1496.

18. Lee Stearns, Victor DeSouza, Jessica Yin, Leah Findlater, and Jon E. Froehlich 2017. Augmented reality magnification for low vision users with the microsoft hololens and a finger-worn camera. ASSETS 2017 - Proceedings of the 19th International ACM SIGACCESS Conference on Computers and Accessibility, ACM Press, 361-362.

19. WHO. 2016. WHO | Visual impairment and blindness. WHO.

20. World Health Organization. 2017. WHO | World Health Organization. WHO.

21. Yan Xu, Maribeth Gandy, Sami Deen, et al. 2008. BragFish: Exploring physical and social interaction in co-located handheld augmented reality games. Proceedings of the 2008 International Conference on Advances in Computer Entertainment Technology, ACE 2008, 276-283.

22. Jing Yang, Yves Frank, and Gábor Soros. 2019. Hearing is believing: Synthesizing spatial audio from everyday objects to users. ACM International Conference Proceeding Series, Association for Computing Machinery, 1-9.

23. Yuhang Zhao, Cynthia L Bennett, Hrvoje Benko, et al. 2018. Enabling People with Visual Impairments to Navigate Virtual Reality with a Haptic and Auditory Cane Simulation. 